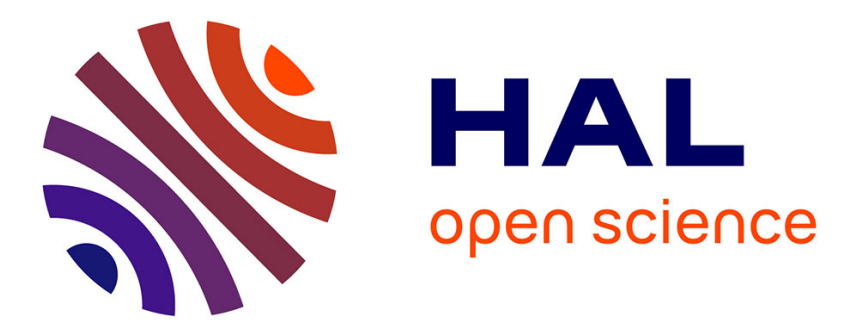

\title{
Multimodal correlative microscopy for in situ detection and quantification of chemical elements in biological specimens. Applications to nanotoxicology
}

Quentin Le Trequesser, Gladys Saez, Marina Simon, Guillaume Devès, Laurent Daudin, Philippe Barberet, Claire Michelet, Marie-Hélène Delville, Hervé Seznec

\section{To cite this version:}

Quentin Le Trequesser, Gladys Saez, Marina Simon, Guillaume Devès, Laurent Daudin, et al.. Multimodal correlative microscopy for in situ detection and quantification of chemical elements in biological specimens. Applications to nanotoxicology. Journal of Chemical Biology, 2015, 8 (4), pp.159-167. 10.1007/s12154-015-0133-5 . hal-01218891

\section{HAL Id: hal-01218891 \\ https://hal.science/hal-01218891}

Submitted on 29 Jan 2021

HAL is a multi-disciplinary open access archive for the deposit and dissemination of scientific research documents, whether they are published or not. The documents may come from teaching and research institutions in France or abroad, or from public or private research centers.
L'archive ouverte pluridisciplinaire HAL, est destinée au dépôt et à la diffusion de documents scientifiques de niveau recherche, publiés ou non, émanant des établissements d'enseignement et de recherche français ou étrangers, des laboratoires publics ou privés. 
1 Multimodal correlative microscopy for in situ detection and quantification of chemical elements in 2 biological specimens. Applications to nanotoxicology.

3

4 Quentin Le Trequesser ${ }^{1,2,3,4}$, Gladys $\mathrm{Saez}^{1,2}$, Marina Simon ${ }^{1,2}$, Guillaume Devès ${ }^{1,2}$, Laurent Daudin ${ }^{1,2}$, Philippe 5 Barberet ${ }^{1,2}$, Claire Michelet ${ }^{1,2}$, Marie-Hélène Delville, ${ }^{3,4}$ and Hervé Seznec ${ }^{1,2^{*}}$

6

$7 \quad{ }^{1}$ Université de Bordeaux, Centre d'Etudes Nucléaires Bordeaux Gradignan (CENBG), Chemin du solarium, F833175 Gradignan, France.

$9 \quad 2$ CNRS, UMR5797, Centre d'Etudes Nucléaires Bordeaux Gradignan (CENBG), Chemin du solarium, F-33175 10 Gradignan, France.

$11{ }^{3}$ CNRS, UPR9048, Institut de Chimie de la Matière Condensée de Bordeaux (ICMCB), 87 avenue du Dr. A. 12 Schweitzer, Pessac, F-33608, France

$13{ }^{4}$ Université de Bordeaux, Institut de Chimie de la Matière Condensée de Bordeaux (ICMCB), 87 avenue du Dr. 14 A. Schweitzer, Pessac, F-33608, France.

15

16 * Corresponding author: Hervé Seznec, herve.seznec@ @enbg.in2p3.fr (phone: +33 557120864). 


\section{Abstract}

2 Correlative microscopy is the application of two or more distinct microscopy techniques to the same region of a

3 sample, generating complementary morphological and structural information that exceeds what is possible with

4 any single technique to answer a biological question. We propose an approach based on a multimodal correlative 5 microscopy, via two imaging and analytical techniques: fluorescence microscopy (FM) and ion beam analysis

6 (IBA) to investigate in vitro nanoparticles (NPs) interactions. Indeed, the explosive growth in Nanotechnology

7 has led to their utilization in a wide range of applications from therapeutics to multimodal imaging labeling.

8 However, the risks for adverse health effects have not been clearly established. Detecting and tracking

9 nanomaterials in biological systems is thus challenging and essential to understand the possible NPs-induced 10 adverse effects. Indeed, assessing in situ NPs internalization at the single cell level is a difficult but critical task 11 due to their potential use in Nanomedicine. One of the main actual challenges is to control the number of NPs internalized per cell. The data obtained by both FM (fluorescence microscopy) and IBA (ion beam analysis)

13 were strongly correlated in terms of detection, tracking, and colocalization of fluorescence and metal detection.

14 IBA not only provides the in situ quantification of exogenous elements in a single cell but also of all the other 15 endogenous elements and the subsequent variation in their cellular homeostasis. This unique property gives 16 access to dose-dependent response analyses and therefore new perspectives for a better insight on the effect of metal oxide NPs on cellular homeostasis.

18

\section{Keywords}

20 Correlative Microscopy, Ion Beam Analysis, Nanoparticles Detection, Single Cell, In Situ Quantification. 
2 Correlative microscopy is usually considered as the application of two or more distinct microscopy techniques to

3 the same region of a defined biological sample, producing complementary morphological, structural and

4 functional information that exceeds what is possible with any single technique to answer a specific biological

5 question (Mironov and Beznoussenko 2009; Caplan et al 2011). The most commonly reliable correlative

6 combination, referred to as "Correlative Light and Electron Microscopy" or CLEM, combines light and

7 transmission electron microscopy. Light microscopy delivers functional information through fluorescent labeling

8 in both living and fixed samples while electron microscopy extends this information through detailed high-

9 resolution images of the same region of interest. Nevertheless, the study of the living is not only limited to

10 genes/proteins/functions and structural information. Indeed, as observed in biological specimens from bacteria to

11 human, metals and trace elements homeostasis play a key role in the cellular regulation physiology. The life of

12 an organism is critically dependent on the proper regulation of uptake, assimilation, intracellular distribution of

13 trace chemical elements, such as ions, metals or exogenous inorganic compounds. These elements, often at the

14 trace level, play a considerable role in physiology and pathology of biological systems. For example, iron is

15 required for oxygen delivery to tissue, the control of cell growth and differentiation and energy metabolism

16 (Andrews 2008; Waldvogel-Abramowski et al 2014). Zinc is involved in immunity, growth and development

17 (Nolte et al 2004), an enzyme cofactor and ensures the correct functioning of over 2000 transcription factors

18 (Takeda 2001; Jeong and Eide 2013). Calcium acts as a crucial second messenger and is involved in various

19 tissular and cellular processes such as muscle contraction (Kurebayashi and Ogawa 2001) and skin homeostasis

20 (Proksch et al 2008; Savignac et al 2014). Thus, the study of the cellular biochemistry in relation to the function

21 and structure in normal or physio-pathological conditions is a critical step toward a complete understanding of

22 the whole cellular metabolic processes. In that sense, it is mandatory to develop imaging and analytical methods

23 to investigate the in situ cellular content, the structural organization, and the function.

24 Only few methods give in situ and quantitative information about the whole composition of a sample. It is thus

25 essential to develop such a method, which considers biological samples in their whole integrity, mass, structure

26 and chemicals (trace elements and ions) and protein contents.

27 With the development of Nanosciences and Nanotechnologies, there is an increasing need in developing such 28 micro-imaging and micro-analytical method. Nanomaterials or nanoparticles (NPs) are defined as objects with at 29 least one of their dimensions ranging from 1 to $100 \mathrm{~nm}$ (Krug and Wick 2011). NPs have unique 30 physicochemical properties such as large surface area per gram, high surface/volume ratio, surface reactivity, 
charge and shape, which make them extensively used in industry. As their size approaches that of proteins, DNA and other biological species, they are used in bio-applications such as therapeutics (Agostinelli et al 2015), antimicrobial agents (Rai et al 2009), transfection vectors (Jong and Borm 2008; Huang et al 2014) and for fluorescent labeling (Le Trequesser et al 2013; Key and Leary 2014). Nevertheless, besides the numerous physical and chemical advantages exhibited by NPs, the risks for adverse health effects due to prolonged or repetitive exposures at various concentration levels in biological species and in the environment have not yet been clearly established. It has been notified by different public authorities and governments worldwide that the current methods for chemical safety assessments have to be modified and reevaluated to address the particular characteristics of NPs and more especially, to assess the biological effects of these highly reactive materials (Yun et al 2009). In particular, the behavior of NPs inside living cells and the associated specific metabolic responses are, to date, not fully understood.

There is a real need for the development of analytical methods, able to in situ detect and quantify NPs, whatever their size, nature and surface reactivity and whether they are native or functionalized.

14 Thus, we have developed an approach based on a correlative microscopy via the complementarity of two imaging and analytical techniques: fluorescence microscopy (FM) and ion beam analysis (IBA) to detect and quantify NPs at the cellular scale of biological models (from human in vitro cells to micro-organisms) and investigate NPs exposure consequences and interactions with the living (Le Trequesser et al 2014a; Le Trequesser et al 2014b). In this article, we will focus on the new possibilities offered by this method in terms of local quantification of particle aggregates at the sub-cellular level.

\section{Results}

22 In order to demonstrate the capability of applying such correlative methods to follow NPs at the cellular level, 23 we exposed cells to a $2 \mu \mathrm{g} \cdot \mathrm{cm}^{2}-\mathrm{TiO}_{2}$ concentration and followed NPs in the living state as well as after the 24 fixation required for IBA analysis. To perform these different types of analyses on a same sample, we developed 25 (i) a sample holder dedicated to these experiments, (ii) sample preparations protocols adapted for multimodal analysis and (iii) methods to precisely retrieve a specific region of interest on the different experimental setups and make the correlation observations faster and easier.

1. Universal sample holder design dedicated to multimodal analysis 
1 We designed a specific sample holder suitable for cell culture, cell handling/preparation on such a holder, and multimodal analysis (Fig. 1a (insert)). It is designed for biological experiments: cell culture, in vitro observations using routine optical microscopy as well as chemical elemental analysis and imaging. This sample holder is made of a PEEK frame covered with a $2 \mu \mathrm{m}$-thick polycarbonate foil. Cells are directly grown on this polycarbonate foil. They are maintained in sterile culture conditions for several days and then used for the different experimental conditions, such as NPs exposure (Le Trequesser et al 2014b). The thin polycarbonate foil gives no $\mathrm{X}$-ray signal during IBA analysis because it contains only $\mathrm{C}, \mathrm{N}$ and $\mathrm{O}$ that produce $\mathrm{X}$-rays that are filtered out by the x-ray detectors. Although polycarbonate foil present local asperities and defaults, it does not preclude its use for cellular observation or impact the quality of cell imaging, using either optical or ion microscopy (Fig. 1a and Fig. 1c).

2. Live imaging, cellular compartments identification and NPs tracking

Fluorescent dye-modified $\mathrm{TiO}_{2}$-NPs $\left(\mathrm{TRITC}-\mathrm{TiO}_{2}-\mathrm{NPs}\right.$ ) were designed, synthesized, and grafted with fluorophores commonly used in biology such as Tetramethyl Rhodamine isothiocyanate (TRITC) (Wessendorf and Brelje 1992). Such a surface chemical modification allows the detection and tracking of the NPs as well as their in situ and in vitro localizations in living cells using FM (Fig. 1b, white arrows). The cell nucleus and specific organelles such as the Golgi apparatus, lysosomes, early and late endosomes were stained using well characterized fluorescent markers, the vital-dye Hoechst ${ }^{33342}$ (blue) and the transduced CellLight ${ }^{\circledR}$ technology (green), respectively. This allowed to assess the intracellular localization of the TRITC-TiO ${ }_{2} \mathrm{NPs}$ (red), 24 hours after exposure (Fig. 1b). NPs were exclusively found in the cytoplasm of exposed cells with no detection in the cell nucleus. Moreover, NPs are randomly localized in the cytoplasm all around the nucleus with a close vicinity to the Golgi apparatus (perinuclear region). NPs can be observed as "free" in the cytoplasm or associated with endosomes (early and late) and lysosomes, in both living and fixed human cells (Fig. 1b, Fig. 2 and Fig. 3). $\mathrm{TiO}_{2}$-NPs displayed the same localization whatever the cell type studied i.e. primary keratinocytes (PHFK) (Fig. 1b) and primary endothelial cells (HUVEC) (Fig. 2 and Fig. 3). The combination of light and fluorescence microscopy can discriminate between dense cellular structures and NPs aggregates that appear in cells as black or red dots, respectively (Fig. 1a and Fig. 1b). Although light microscopy is very informative about the localization of NPs inside exposed cells, it is still not possible to evaluate the exact number of NPs per cell. The main difficulty concerning the quantification of NPs using fluorescence microscopy mainly results from the 
experiment. Because they are directly sensitive to the NPs with no need of an intermediate signal like fluorescence of a grafted molecule, IBA methods are interestingly complementary to more conventional optical microscopy. Furthermore, they can also be quantitative, giving access to information about the intracellular biochemical content that is usually unknown.

3. Cryofixation of cells after live imaging

The main constraint in performing ion microscopy is the necessity to perform analysis under vacuum condition. For this reason, we had to develop a protocol for cell fixation enabling to preserve its ultrastructure and its biochemical integrity. Chemical fixation is known to modify the chemical composition of cells by replacement of their cellular medium with a polymer aiming to preserve cellular ultrastructure. At the same time, the evacuation of water releases free ions and species thus modifying its composition. This is the reason why it is appropriate to give priority to physical fixation methods like cryogenic ones. These cryogenic procedures provide rapid cessation of the cellular activity within the millisecond time scale (as compared to several seconds for conventional fixation) (Carmona et al 2008). For sample preparation, we therefore established a two-step protocol in which LM investigations of living cells were performed first, followed by a plunge freezing fixation and dehydration at low temperature without addition of any fixative compounds (see Material and methods).

4. IBA microscopy and quantification of nanoparticles at cellular scale

After cryofixation and lyophilisation, the samples were analyzed by IBA to obtain precise quantitative data on their elemental chemical composition. Contrast in Scanning Transmission Ion Microscopy ( $\mu$-STIM) images is due to local differences in density and allow the detection of cell structures such as nucleolus and cytoplasm (Fig. 1c) (Barberet et al 2011). Comparing Figures 1a and 1c, the nucleolus can be easily detected suggesting that this nuclear region is dense. In the cytoplasm, "vacuoles" can be easily defined from both in vitro analysis and cryofixed sample. Unfortunately, NPs suggested as dark dots on phase contrast imaging (Fig. 1a) could not always be seen using $\mu$-STIM rendering. This is because STIM contrast is not specific of NPs but to average local thickness. As cells are seen through the polycarbonate foil, changes in support thickness or density are liable to confusion with cellular structures. This limit can eventually be compensated by a better spatial resolution (100-200 nm) of the beam compared to optical microscopy (200-400 nm). 
1 the proton beam, the chemical elements present in the cells, follow a schematic excitation-desexcitation process that eventually ends up with the emission of a photon with an energy characteristic of the atomic number of the excited element. The sum of all the emitted photon events builds a characteristic peak spectrum (Fig. 4), usually considered as a chemical fingerprint of the sample. The usual experimental setups and detectors used for PIXE experiments allow quantifying at the same time all the elements heavier than $\mathrm{Na}$ with a 1 to $10 \mu \mathrm{g} . \mathrm{g}^{-1}$ of dry mass detection limit. Accuracy in measuring elements is usually driven by charge collection and detector efficiencies and is about $20 \%$.

8 We used ion microscopy to observe the distribution of cellular elements like phosphorus and potassium and to quantify the intracellular amount of $\mathrm{TiO}_{2} \mathrm{NPs}$ with titanium mapping. Chemical element maps are computed after sorting photons according to the beam position at the time of recording and using an energy window centered around a specific element (Fig. 4) Maps usually represent the number of detected events at the beam position and can be quantitative; noises and background can be filtered out. Inversely, chemical element maps can be sorted in order to retrieve the local PIXE spectrum required for quantification.

14 Here, phosphorus is found homogenously distributed in the cell with a higher concentration in the nuclear area

(Fig. 1d and Fig. 2d). Potassium (Fig. 1e) and sulfur (Fig. 2e) are homogeneously distributed in the cytoplasm. Titanium is located in the cytoplasmic perinuclear region as aggregates (Fig. 1f and Fig. 2f), as previously observed using LM and FM (Fig. 1a and Fig. 1b). NPs displayed the same perinuclear localization whatever their surface state i.e. functionalized (Fig. 2a) or native (Fig. 2f). The average content of titanium in the cell presented here is very low (5.6 ng.cm-2) compared to the $2 \mu \mathrm{g} \mathrm{cm}{ }^{-2}$ exposure dose of the cell population (Fig. 4). In the aggregates found around the nucleus, the number of particles varies from 200 NPs to 9,000 NPs, giving locally exposure dose as much as twice the average exposure dose (Fig. 5). In the meantime, no trace of titanium was detected in control cells confirming that the titanium distribution observed by $\mu$-PIXE has to be attributed to the $\mathrm{TiO}_{2}$ NPs (Fig. 1f, white arrows) and in good agreement with our previous observations by conventional FM (Fig. 1b). In addition, $\mu$-PIXE reveals additional NPs sites of accumulation not detected by FM. The cellular internalization of NPs has already been shown by high-resolution 3D imaging using confocal microscopy with fluorescent modified NPs and TEM analysis on ultra-thin sections (Simon et al 2011; Stefančíková et al 2014).

\section{Discussion}

29 IBA can provide useful information that other imaging techniques are not able to do, especially at the subcellular level. Indeed, beyond its imaging ability, IBA also provides a quantification of chemical elements 
constituting the sample under study, whatever its nature. In this case, it can analyze biological samples and focus on a chosen region of interest of a single cell. Its combination with other techniques provides both a morphological cellular imaging and precise quantitative data on the elemental chemical composition of the sample. These techniques present the following advantages (i) a sample preparation which does not require chemical fixation, staining nor sectioning; (ii) large observable areas with the option to focus on a given region of interest (ROI) of the sample; (iii) the quantification of the chemical elements content with a sensitivity of a few $\mu \mathrm{g}$ per gram $\left(\mu \mathrm{g} \cdot \mathrm{g}^{-1}\right)$; (iv) the analysis of the overall cell volume with a sensitivity to cell density changes which allows the identification of not only the different cell compartments (nucleus, nucleolus, cytoplasm), but also any dense structure with a size comparable to that of the beam diameter (about $300 \mathrm{~nm}$ spatial resolution). As shown in the example here, this ability to observe and quantify NPs within individual cells allows addressing the particular questions of bio-accumulation and bio-persistence of endogenous/exogenous chemical elements such as metal oxide NPs.

This is a crucial challenge for further applications in biomedicine. The accurate determination of dose when studying NPs internalization in cells is essential for quantitative NPs toxicology and pharmacology. Up to now, most of the known methods cannot accurately reflect the concentration of internalized NPs in a single cell. There are numerous analytical tools, including microscopy, mass spectrometry (MS), inductively coupled plasma MS (ICPMS), liquid chromatography MS (LC-MS) and radioactive isotope which are used to estimate the cellular dose of NPs but provide information at a macroscopic level. None of them can provide as IBA techniques a precise identification of cellular dose as well as distribution giving access to a further systematic consideration of dose-response assessment. Moreover, many inorganic NPs suffer from a lack of fluorescence in the optical regime and cannot be followed by light microscopy in their native state. To overcome this point, NPs are often functionalized with fluorescent dyes but (i) quantification remains difficult because the level of tagging of a single NP is usually unknown, and (ii) the chemical modification of the surface of a NP may alter its cellular distribution as compared to that of a bare one.

26 This work highlighted the suitability of using IBA with other techniques in a correlative study concerning the 27 future assessments of NPs interactions with living cells. The correlative approach gives information on the 28 impacts of these NPs in terms of detection, identification, localization and quantification at the single cell level of both native and chemically modified NPs. Cells were grown on a single specific sample holder allowing the 
successive in vitro analysis of human cells (PHFK and HUVEC) exposed to $\mathrm{TiO}_{2} \mathrm{NPs}$ with FM and IBA. The set-up allowed a dual analysis with in vitro and cryofixed images of the same set of cells. The procedure used to prepare the samples was chosen as to avoid the resin embedding thus allowed to study by IBA the ion homeostasis alteration due to the exposure to NPs. Both techniques, FM and IBA, gave corroborative results showing that the fluorescent functionalization of NPs to see them is not a mandatory process and that any sample can be observed whatever its state surface. This then opens the field to toxicological study on native NPs (not functionalized). IBA main interest here is that it provides critical data concerning the local quantification of all the chemical elements including exogenous ones (metals and metal oxides) when present in regions of interest. As such, this specific technique may become a much useful tool as compared to more classical approaches for two reasons: (i) the latter are based on chemical analysis and mass spectrometry which provide information at the macroscopic level; (ii) IBA can provide new reply to the problematic of dose-dependent response analyses and a better understanding of metal oxide NPs toxicity by a follow-up of the concentration of endogenous elements.

\section{Materials and Methods}

16 Synthesis of fluorescent dye-modified $\mathrm{TiO}_{2}$-NPs. $\mathrm{TiO}_{2} \mathrm{P} 25 \mathrm{AEROXIDE}^{\circledR}$ nanoparticles kindly provided by Degussa/Evonik consist in a mixture of anatase $(80 \%)$ and rutile $(20 \%)$ phases (specific surface area of $55 \mathrm{~m}^{2} \cdot \mathrm{g}^{-}$ ', $25 \mathrm{~nm} \pm 10 \mathrm{~nm}$ ). Their surface modification was performed according to published procedure (Simon et al., 2011). Briefly, $600 \mathrm{mg}$ of $\mathrm{TiO}_{2}$-NPs were mixed with $3 \mathrm{~mL}$ of ammonium hydroxide $25 \%$ (v/v), $100 \mu \mathrm{L} \mathrm{3-}$ aminopropyltriethoxysilane and absolute ethanol and stirred at room temperature for $48 \mathrm{~h}$. The suspension was then heated at $100{ }^{\circ} \mathrm{C}$ for $2 \mathrm{~h}$ under reflux. The white powder was then washed 5 times with absolute ethanol. The powder was then added to a $30 \mathrm{~mL}$ of $\mathrm{Na}_{2} \mathrm{CO}_{3}(0.01 \mathrm{M})$ aqueous solution containing $2.5 \mathrm{mg}$ of tetramethylrhodamine-isothiocyanate (TRITC) and stirred for $48 \mathrm{~h}$. NPs were finally washed several times with a $\mathrm{Na}_{2} \mathrm{CO}_{3}(0.01 \mathrm{M})$ solution and then with milliQ water.

Cell exposure. Primary human foreskin keratinocytes (PHFK) or human umbilical vein endothelial cells (HUVEC), derived from normal human tissue, were obtained from Invitrogen (Cergy Pontoise, France). They were respectively grown in Defined Keratinocyte-SFM or M200 complemented with LSGS (Gibco, Life Technologies) with $100 \mathrm{mg} \cdot \mathrm{mL}^{-1}$ penicillin/streptomycin at $37^{\circ} \mathrm{C}$ in a $5 \%(\mathrm{v} / \mathrm{v}) \mathrm{CO}_{2}$ humidified atmosphere. Passages are realized at $80 \%$ confluency. The suspensions of fluorescent dye-modified $\mathrm{TiO}_{2}$-NPs were prepared 
hereby known as "stock suspensions". $\mathrm{TiO}_{2}$-NPs were dispersed by high intensity sonication at room temperature (RT) using a probe for $1 \mathrm{~min}$ before cell exposure. Stock suspensions were diluted at the appropriate concentration in defined culture medium in order to obtain exposure suspension at $2 \mu \mathrm{g} . \mathrm{cm}^{-2}$ (final concentration).

Labeling of cellular organelles and nucleus of PHFK and HUVEC. Before NPs exposure, cells were exposed to the CellLight ${ }^{\circledR}$ BacMan reagent of interest $(40 \mu \mathrm{L})$ as recommended by the suppliers (Life Technologies). Here, we used CellLight ${ }^{\circledR}$ BacMan reagent to label specific organelles such as the Golgi apparatus, lysosomes, early and late endosomes. CellLight ${ }^{\circledR}$ technology is a modified baculovirus, non-replicating in mammalian cells, and expressing a fusion construct of proteins targeted to specific intracellular structures and a green fluorescent protein (GFP). After the desired time of incubation with $2 \mu \mathrm{g} . \mathrm{cm}^{-2}$ fluorescent dye-modified $\mathrm{TiO}_{2}-\mathrm{NPs}$, cells were rinsed 3 times with fresh culture medium.

12 For confocal microscopy acquisitions, cells were fixed with $2 \%(w / v)$ paraformaldehyde (Sigma,St QuentinFallavier, France) in PBS (pH 7.4, without $\mathrm{Ca}^{2+}$ and $\mathrm{Mg}^{2+}$, Invitrogen, Cergy Pontoise, France) for 30 minutes at $4^{\circ} \mathrm{C}$. Then, a cell permeabilization was performed using a solution containing $0.2 \%(\mathrm{v} / \mathrm{v})$ Triton X-100 (Sigma, St Quentin Fallavier, France) in PBS for 15 min. Cells were then rinsed 3 times with PBS and nuclei stained with $10 \mu \mathrm{M}$ Hoechst ${ }^{33342}$ in PBS. Hoechst ${ }^{3342}$ is a vital DNA stain that binds preferentially to A-T base-pairs. Slides were mounted using Prolong Gold Antifade Reagent (Invitrogen, Cergy Pontoise, Fance) and visualized on Leica DMRE TCS SP2 AOBS confocal microscope.

For correlative microscopy experiments, cell culture medium was then supplemented with Hoechst ${ }^{3342}$ at a final concentration of $10 \mu \mathrm{M}$. The cells require no permeabilization for labeling, but do require good physiologic conditions since the dye internalization is an active process. Cells were then incubated at $37{ }^{\circ} \mathrm{C}$ for $1 \mathrm{~h}$ and rinsed 3 times with fresh medium to remove any excess of Hoechst ${ }^{33342}$. The cells were then observed before and after freeze-drying using an optical microscope Zeiss AxioObserver Z1 (Carl Zeiss MicroImaging, GmbH). EC Plan-Neofluar 20x/0.50 Ph2 M27 objective was used for phase contrast and fluorescence imaging. Fluorescence imaging was performed with different excitation/emission filters according to the considered fluorochrome (GFP, Rhodamine, Hoechst ${ }^{3334}$ ).

Cell preparation for high-resolution ion beam microanalysis (IBA). Cells were cultured directly onto ion beam microprobe sample holders as adapted from previous studies. This sample holder is composed of a $2 \mu \mathrm{m}$-thick polycarbonate films tightly stuck on a PEEK support (PolyEther Ether Ketone). Polycarbonate film was chosen for its biocompatibility and mechanical properties. In addition, this polymer is transparent and shows high 
resistance to ion beam without stopping it. PEEK, a colorless thermoplastic organic polymer, resistant to thermal degradation (suitable for numerous cycles of sterilizations by autoclaving) as well as to attack by both organic and aqueous environments, is very robust and widely used in bio-medical applications (medical implants). The polycarbonate film was chosen for its biocompatibility and for its ability to resist to different protocols and analytical constraints. In addition, polycarbonate is a transparent polymer, which also shows a high resistance to ion beam without stopping it. The choice of the support in PEEK was essential to allow multi-treatments and multi-analyses on the same sample without degradation. Briefly, cells were directly grown at high confluence on $2 \mu$ m-thick polycarbonate foil for 24 hours in appropriate culture medium, and then exposed to $\mathrm{TiO}_{2}-\mathrm{NPs}_{\text {s }}$ for 24 h. Control cells were prepared similarly with no addition of $\mathrm{TiO}_{2}$-NPs. Cells were rinsed twice in culture medium, rinsed in phosphate saline buffer (PBS) and very briefly rinsed in ultrapure water to remove excess of extracellular salts from PBS. Finally, cells were plunge-freezed at $-120{ }^{\circ} \mathrm{C}$ into liquid nitrogen chilled isopentane, and freeze-dried at $-35^{\circ} \mathrm{C}$ for $96 \mathrm{~h}$.

Single cell recognition during multimodal analyses. Correlative microscopy is limited by the ability to perform single cell recognition and identification in the separate microscopes (Liv et al 2013). Considering the size of a cell, relocating a sample requires a precision of a few tens of micrometers or below. The use of the same microscope stage for the different analysis would in principle solve this problem, but this is not possible because each microscope has different requirements in terms of dimensions, positioning, vacuum, alignment, etc. For this reason, using a coordinate system relative to the sample holder is more appropriate. In our case, living cells were observed and their position recorded using images of the complete sample holder $\left(5 \times 5 \mathrm{~mm}^{2}\right)$ by $\mathrm{LM} / \mathrm{FM}$ microscopy. This was possible using a motorized microscope stage (AxioObserver Z1, Carl Zeiss

21 MicroImaging, $\mathrm{GmbH}$ ) that enables fast tiled acquisition and provides stitching procedures for post-acquisition large-scale image reconstruction. In such a way, cells of interest could be identified individually either relative to any other cell or to the frame. The operation was repeated after freeze-drying in order to maximize our ability to retrieve cells because lyophilization modifies cell opacity. The interest of imaging the entire cell frame is that it enables to retrieve cells on any type of microscope. On the nuclear microprobe set-up, a procedure of alignment of the ion beam with the online visible light microscope is performed with a motorized sample stage with submicrometer accuracy in sample positioning. This enables to spot any cell either by moving the sample or by moving the beam.

Ion beam microanalysis (IBA). Chemical element imaging was carried out at the microprobe beam line of AIFIRA using complementary ion beam analytical techniques ( $\mu$-PIXE, $\mu$-RBS and $\mu$-STIM). The facility is 
1 based on a 3.5 MV (HVEE, The Netherlands) Singletron particle accelerator delivering light ion beams in the

$2 \mathrm{MeV}$ energy range. For Scanning Transmission Ion Microscopy ( $\mu$-STIM), a $2 \mathrm{MeV} \mathrm{He}^{+}$beam was used as a 3 probe with a size in the focal plane around $300 \mathrm{~nm}$ in diameter and at low fluence (2000 ions. $\left.\mathrm{s}^{-1}\right)$. The energy of 4 the transmitted ions is measured with a planar silicon detector (Canberra PIPS detector, $25 \mathrm{~mm}^{2}, 12 \mathrm{keV}$ energy 5 resolution @5.5 MeV), placed at $0^{\circ}$ on the incoming beam axis. For $\mu$-PIXE and $\mu$-RBS, a $1.5 \mathrm{MeV} \mathrm{H}^{+}$ 6 microbeam, focused down to a diameter of about $1 \mu \mathrm{m}$, was scanned over areas of interest using a fast 7 electrostatic scanning system. Measurements on narrow areas containing a limited number of cells (usually 1 to 8 10) were performed. This spatial resolution enabled to discriminate the cytosol and nuclear areas of the cells.. In 9 the case of $\mu$-PIXE, typical beam currents in the range of 50-150 pA were used. Induced X-rays emitted from 10 light ions present in the sample (from $\mathrm{Na}$ to $\mathrm{Ti}$ ) were collected by a high-resolution $\mathrm{Si}$ ( $\mathrm{Li}$ ) solid-state detector

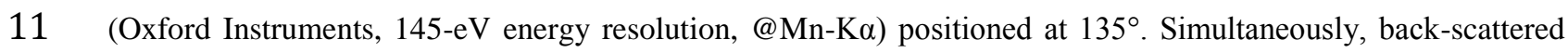
12 protons were detected at $-135^{\circ}$ by a silicon detector (Canberra, partially depleted detector, $25 \mathrm{~mm}^{2}, 12 \mathrm{keV}$ 13 resolution@ $4.5 \mathrm{MeV}$ ). To provide elemental maps, $256 \times 256$ pixels matrices were calculated from $\mu$-PIXE 14 measurements. A simple energy windowing was used to select the X-ray lines and construct yield maps from 15 events stored in the list files. For the extraction of quantitative results, X-rays ( $\mu$-PIXE) and backscattered 16 particles energy spectra (RBS) were obtained from the regions of interest. This operation was carried out using 17 the SupaVISIO software. X-ray spectra were fitted using the Gupix software and the RBS spectra were treated 18 using the SIMNRA software. 
2 The manuscript was written through contributions of all authors. All authors have given approval to the final

3 version of the manuscript.

Funding Sources

6 French National Research Agency supports the research program TITANIUMS (ANR Contaminants, 7 Ecosystèmes, Santé 2010, under the contract n ${ }^{\circ}$ ANR-10-CESA-009). The CNRS and the European Community

8 as an Integrating activity Support "Support of Public and Industrial Research Using Ion Beam Technology

9 (SPIRIT)" under the EC contract $\mathrm{n}^{\circ} 227012$ support the research program. The Region Aquitaine supports the research program TOXNANO. The C'NANO Grand Sud Ouest and the Region Aquitaine support the AIFIRA 11 facility.

\section{Acknowledgment}

14 This research was undertaken on the high-resolution microbeam line at the AIFIRA facility (Applications 15 Interdisciplinaires des Faisceaux d'Ions en Région Aquitaine). We wish to thank the technical staff members of 16 the AIFIRA facility ( $\mathrm{Ph}$. Alfaurt, S. Sorieul). The Région Aquitaine supported financially the AIFIRA facility and the technical development of the microbeam line. The authors acknowledge the Evonik (Degussa) Company (Düsseldorf, Germany) for their generous gift ( $\mathrm{P} 25 \mathrm{TiO}_{2} \mathrm{NPs}$ ). This work has been partly supported by the European Community as an Integrating Activity "Support of Public and Industrial Research Using Ion Beam Technology (SPIRIT)" under the EC contract n 227012. The CNRS and the French National Research Agency (ANR CES2010, TITANIUMS) support the research program. 


\section{Figures Captions}

4

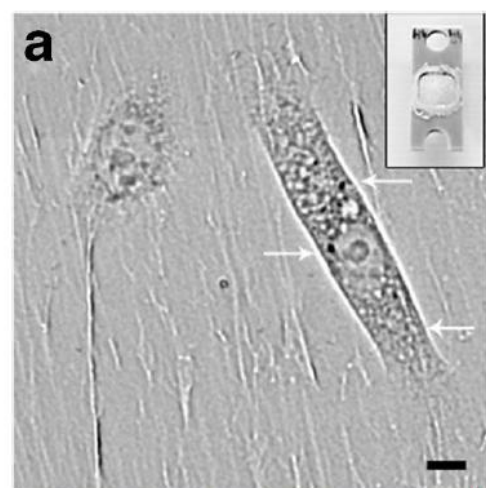

\section{b}
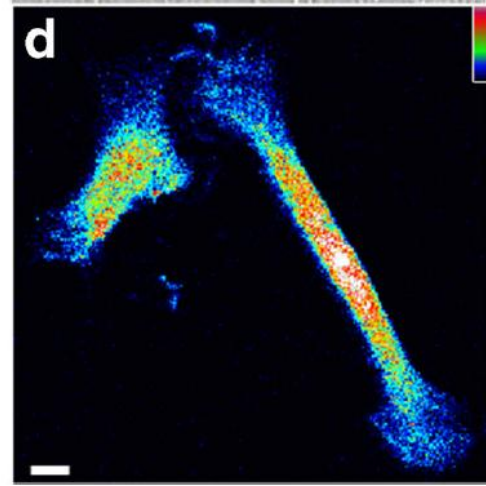

$\boldsymbol{e}$
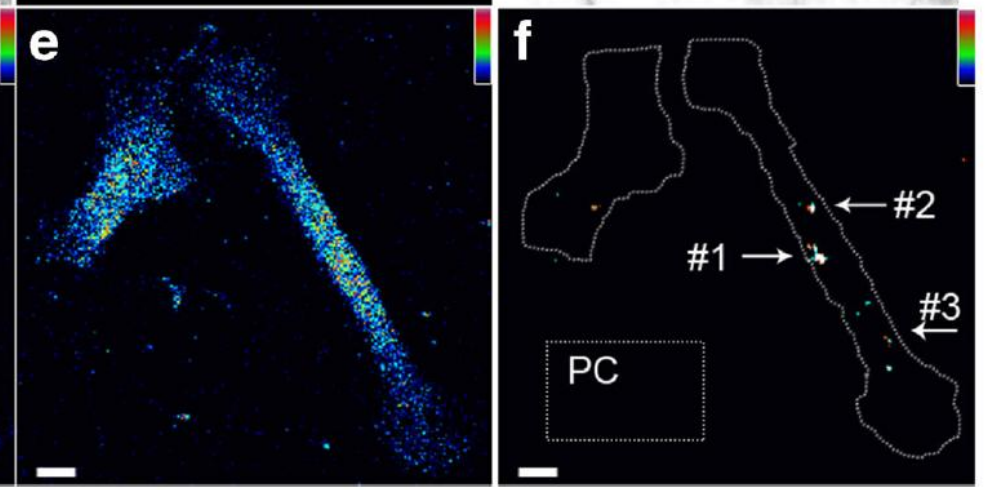

6 Fig. 1 Correlative microscopy: from live in vitro microscopy to IBA. (a) Bright field image of primary

7 keratinocytes plated on $2 \mu \mathrm{m}$-thick polycarbonate foil, (insert: picture of the sample holder) (b) in vitro

8 fluorescence imaging of TRITC- $\mathrm{TiO}_{2}$ NPs loaded cells. Hoechst ${ }^{33342}$ (blue) counterstains DNA. TRITC-TiO 2

9 NPs are shown in red and the Golgi apparatus (CellLight@Golgi-GFP) is displayed in green. (c) $\mu$-STIM

10 performed on the same freeze-dried cells once cryofixed and lyophilized. Density scale ranges from white

11 (minimum) to black (maximum). (d, e, f) $\mu$-PIXE maps of phosphorus, potassium and titanium, respectively.

12 The arrows locate the NPs under the different techniques. Element yields shown in pseudo-color ranging from

13 black (minimum) to pale gray (maximum). Scale bars: $10 \mu \mathrm{m}$. 


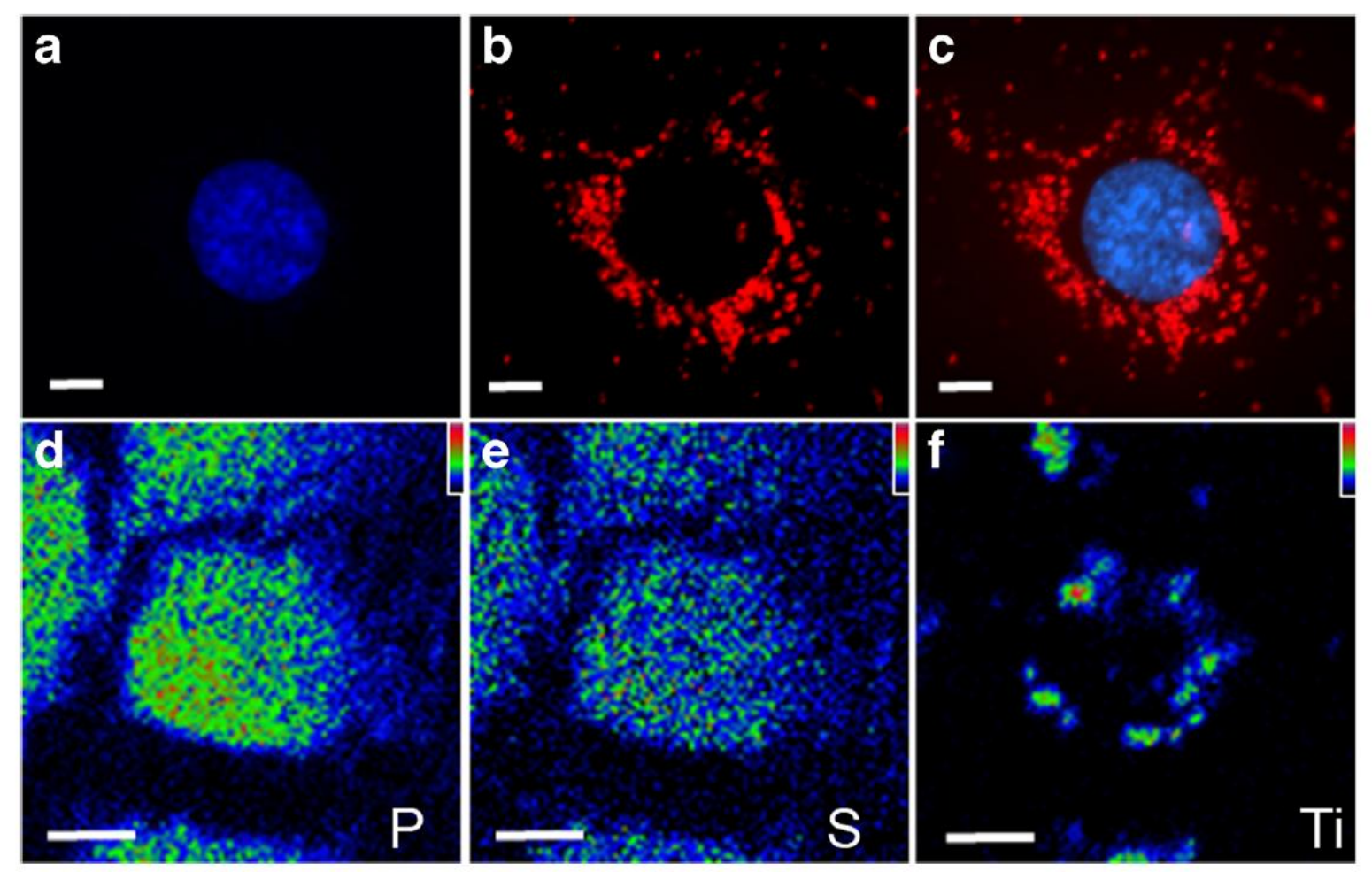

2 Fig. 2 HUVEC cells exposed to $2 \mu \mathrm{g} . \mathrm{cm}^{-2}$ of $\mathrm{TiO}_{2} \mathrm{NPs}(\mathbf{a}, \mathbf{b}, \mathbf{c})$ Confocal fluorescence imaging of loaded cells.

3 Hoechst $^{33342}$ (blue) counterstains DNA and TRITC-TiO ${ }_{2}$ NPs are shown in red. (d, e, f) $\mu$-PIXE maps of

4 phosphorus, sulfur and titanium elements, respectively. The two microscopic techniques revealed an exclusive

5 perinuclear localization of the $\mathrm{TiO}_{2}$-NPs. Element yields shown in pseudo-color ranging from black (minimum)

6 to pale gray (maximum). Scale bars: $10 \mu \mathrm{m}$.

7 


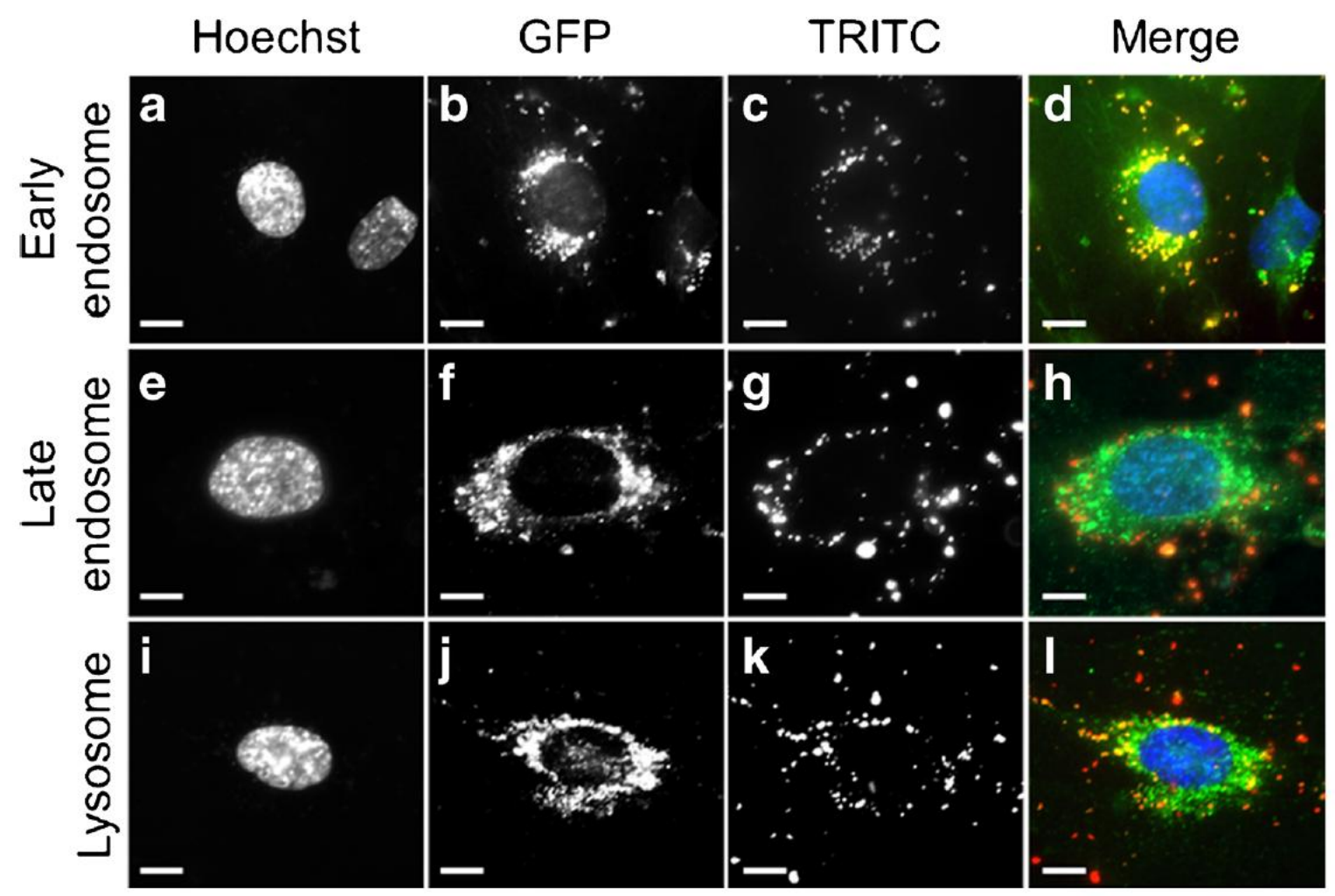

2 Fig. 3 TRITC-TiO $\mathrm{NPs}_{2}$ subcellular localization. HUVEC cells were transduced to label (a-d) early endosomes,

3 (e-h) late endosomes and (i-l) lysosomes (green staining). Hoechst ${ }^{33342}$ (blue) counterstains DNA and TRITC-

$4 \mathrm{TiO}_{2} \mathrm{NPs}$ are shown in red. TRITC-TiO 2 NPs display no specific localization in the different organelles tested.

5 NPs associated with the considered tagged organelles are depicted in yellow, "free" NPs are detected as red dots.

6 Scale bars: $10 \mu \mathrm{m}$.

7 


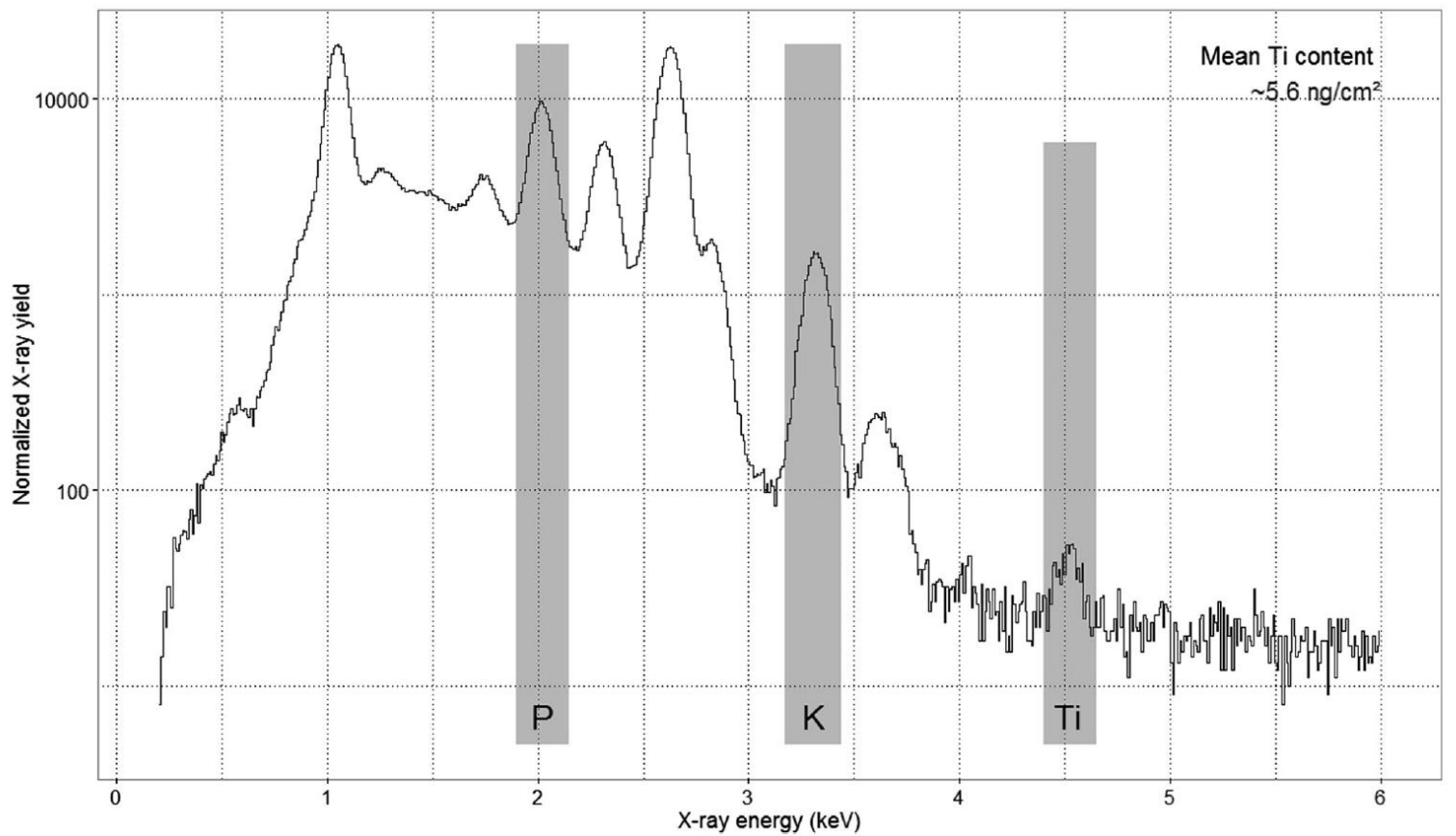

2 Fig. 4 Single cell $\mu$-PIXE spectrum corresponding to cell showed in fig. 1 (right). Energy windows (grays)

3 around peaks of phosphorus, potassium and titanium were used to compute the distribution of elements shown

4 on fig. 1d, 1e and 1f. Quantification of elements indicates that the average Ti content in this cell is very low 5 compared to average exposure of cells $\left(2 \mu \mathrm{g} \cdot \mathrm{cm}^{-2}\right)$.

6

7 

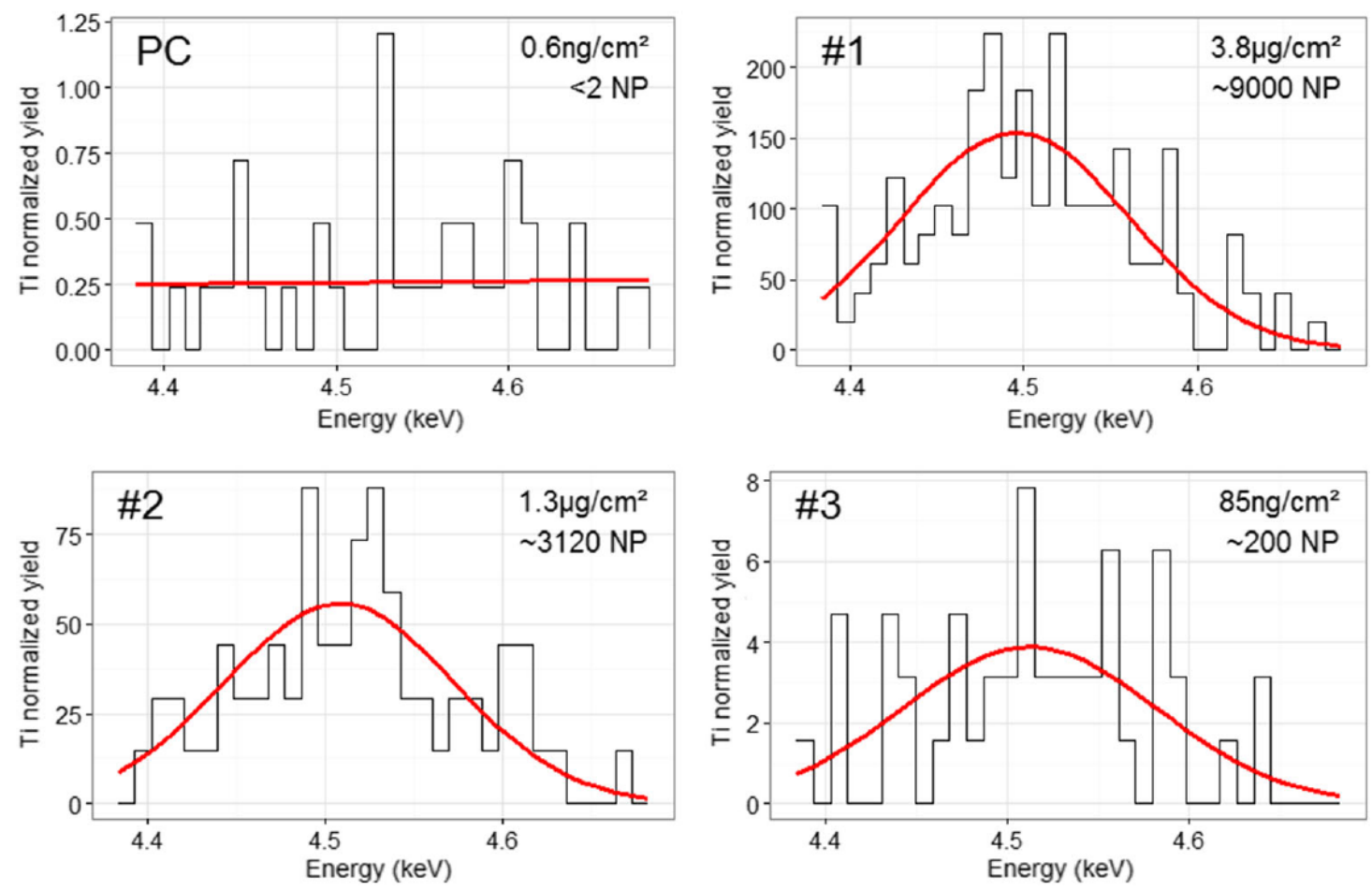

2 Fig. 5 Ti peaks extracted from regions of interest on Ti map (Fig. 1f) corresponding to polycarbonate support (PC) taken outside the cell of interest and cellular aggregates \#1, \#2 and \#3 as shown in Fig. 1f. Peak yield fitting (red) enables to convert peak intensity into Ti concentration and calculate the equivalent number of NPs present in the dedicated region of interest. 
Bibliography

Agostinelli E, Vianello F, Magliulo G, et al (2015) Nanoparticle strategies for cancer therapeutics: Nucleic acids, polyamines, bovine serum amine oxidase and iron oxide nanoparticles (Review). Int J Oncol 46:5-16. doi: 10.3892/ijo.2014.2706

Andrews NC (2008) Forging a field: the golden age of iron biology. Blood 112:219-30. doi: 10.1182/blood2007-12-077388

Barberet P, Daudin L, Gordillo N, et al (2011) First results obtained using the CENBG nanobeam line: Performances and applications. Nucl Instruments Methods Phys Res Sect B Beam Interact with Mater Atoms 269:2163-2167. doi: 10.1016/j.nimb.2011.02.036

Caplan J, Niethammer M, Taylor RM, Czymmek KJ (2011) The power of correlative microscopy: multi-modal, multi-scale, multi-dimensional. Curr Opin Struct Biol 21:686-93. doi: 10.1016/j.sbi.2011.06.010

Carmona A, Devès G, Ortega R (2008) Quantitative micro-analysis of metal ions in subcellular compartments of cultured dopaminergic cells by combination of three ion beam techniques. Anal Bioanal Chem 390:158594. doi: 10.1007/s00216-008-1866-6

Huang X, Shen S, Zhang Z, Zhuang J (2014) Cross-linked polyethylenimine-tripolyphosphate nanoparticles for gene delivery. Int J Nanomedicine 9:4785-94. doi: 10.2147/IJN.S61910

Jeong J, Eide DJ (2013) The SLC39 family of zinc transporters. Mol Aspects Med 34:612-9. doi: 10.1016/j.mam.2012.05.011

Jong W De, Borm P (2008) Drug delivery and nanoparticles: applications and hazards. Int J Nanomedicine 3:133-149.

Key J, Leary J (2014) Nanoparticles for multimodal in vivo imaging in nanomedicine. Int J Nanomedicine 711 726.

Krug HF, Wick P (2011) Nanotoxicology: an interdisciplinary challenge. Angew Chem Int Ed Engl 50:1260-78. doi: 10.1002/anie.201001037

Kurebayashi N, Ogawa Y (2001) Depletion of Ca2+ in the sarcoplasmic reticulum stimulates Ca2+ entry into mouse skeletal muscle fibres. J Physiol 3:185-199.

Le Trequesser Q, Devès G, Saez G, et al (2014a) Single cell in situ detection and quantification of metal oxide nanoparticles using multimodal correlative microscopy. Anal Chem 86:7311-9. doi: 10.1021/ac501318c

Le Trequesser Q, Saez G, Devès G, et al (2014b) In situ titanium dioxide nanoparticles quantitative microscopy in cells and in C. elegans using nuclear microprobe analysis. Nucl Instruments Methods Phys Res Sect B Beam Interact with Mater Atoms 341:58-64. doi: 10.1016/j.nimb.2014.06.031

Le Trequesser Q, Seznec H, Delville M-H (2013) Functionalized nanomaterials: their use as contrast agents in bioimaging: mono- and multimodal approaches. Nanotechnol Rev. doi: 10.1515/ntrev-2012-0080

Liv N, Zonnevylle a C, Narvaez AC, et al (2013) Simultaneous correlative scanning electron and high-NA fluorescence microscopy. PLoS One 8:e55707. doi: 10.1371/journal.pone.0055707

Mironov AA, Beznoussenko G V (2009) Correlative microscopy: a potent tool for the study of rare or unique cellular and tissue events. J Microsc 235:308-21. doi: 10.1111/j.1365-2818.2009.03222.x

Nolte C, Gore A, Sekler I, et al (2004) ZnT-1 expression in astroglial cells protects against zinc toxicity and slows the accumulation of intracellular zinc. Glia 48:145-55. doi: 10.1002/glia.20065 
Proksch E, Brandner JM, Jensen J-M (2008) The skin: an indispensable barrier. Exp Dermatol 17:1063-1072. doi: $10.1111 / \mathrm{j} .1600-0625.2008 .00786 . \mathrm{x}$

Rai M, Yadav A, Gade A (2009) Silver nanoparticles as a new generation of antimicrobials. Biotechnol Adv 27:76-83. doi: 10.1016/j.biotechadv.2008.09.002

Savignac M, Simon M, Edir A, et al (2014) SERCA2 dysfunction in Darier disease causes endoplasmic reticulum stress and impaired cell-to-cell adhesion strength: rescue by Miglustat. J Invest Dermatol 134:1961-70. doi: 10.1038/jid.2014.8

Simon M, Barberet P, Delville M-H, et al (2011) Titanium dioxide nanoparticles induced intracellular calcium homeostasis modification in primary human keratinocytes. Towards an in vitro explanation of titanium dioxide nanoparticles toxicity. Nanotoxicology 5:125-139.

Stefančíková L, Porcel E, Eustache P, et al (2014) Cell localisation of gadolinium-based nanoparticles and related radiosensitising efficacy in glioblastoma cells. Cancer Nanotechnol 5:6. doi: 10.1186/s12645-0140006-6

Takeda A (2001) Zinc homeostasis and functions of zinc in the brain. Biometals 14:343-51.

Waldvogel-Abramowski S, Waeber G, Gassner C, et al (2014) Physiology of iron metabolism. Transfus Med hemotherapy Off Organ der Dtsch Gesellschaft für Transfusionsmedizin und Immunhamätologie 41:21321. doi: $10.1159 / 000362888$

Wessendorf MW, Brelje TC (1992) Which fluorophore is brightest? A comparison of the staining obtained using fluorescein, tetramethylrhodamine, lissamine rhodamine, Texas red, and cyanine 3.18. Histochemistry 98:81-5. 